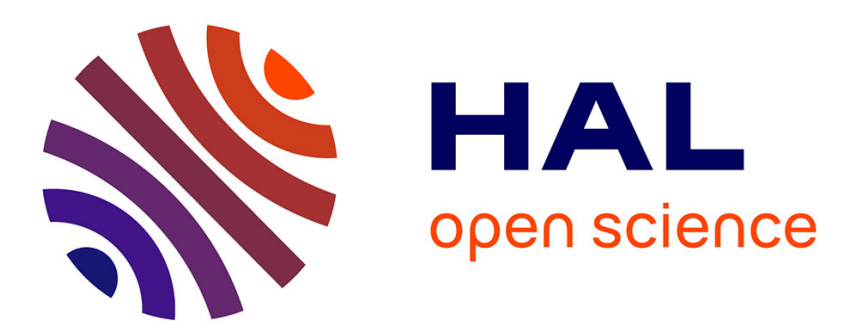

\title{
Réalisation d'une machine de fluage biaxiale (traction-torsion) à contraintes constantes
}

\author{
P. Delobelle, D. Varchon
}

\section{To cite this version:}

P. Delobelle, D. Varchon. Réalisation d'une machine de fluage biaxiale (traction-torsion)

à contraintes constantes. Revue de Physique Appliquée, 1983, 18 (10), pp.667-674. 10.1051/rphysap:019830018010066700 . jpa-00245129

HAL Id: jpa-00245129

https://hal.science/jpa-00245129

Submitted on 1 Jan 1983

HAL is a multi-disciplinary open access archive for the deposit and dissemination of scientific research documents, whether they are published or not. The documents may come from teaching and research institutions in France or abroad, or from public or private research centers.
L'archive ouverte pluridisciplinaire HAL, est destinée au dépôt et à la diffusion de documents scientifiques de niveau recherche, publiés ou non, émanant des établissements d'enseignement et de recherche français ou étrangers, des laboratoires publics ou privés. 


\title{
Réalisation d'une machine de fluage biaxiale (traction-torsion) à contraintes constantes $\left(\sigma_{z z}, \sigma_{z \theta}\right)$
}

\author{
P. Delobelle et D. Varchon \\ Laboratoire de Mécanique Appliquée, LA 004, Faculté des Sciences et des Techniques, Route de Gray, \\ 25030 Besançon Cedex, France
}

(Reçu le 30 mai 1983, révisé le 8 juillet, accepté le 12 juillet 1983)

\begin{abstract}
Résumé. - On présente une machine de fluage de grande rigidité permettant de réaliser des essais biaxiaux à haute température sous contraintes constantes (traction $\sigma_{z z}$, torsion $\sigma_{z \theta}$ ). La force axiale est obtenue à l'aide de poids morts et l'asservissement de la contrainte correspondante est réalisée par l'intermédiaire d'une came faisant varier le moment du point d'application de la force en fonction de l'allongement de l'éprouvette. Le couple de torsion est obtenu à l'aide de moteurs couples directement montés en série sur le mors de torsion et dont l'intensité d'alimentation suit la loi d'asservissement à réaliser. Cette solution permet également de réaliser des essais sous contrainte cyclique de torsion, la contrainte axiale de polarisation étant fixe.

Les performances mécaniques de cette machine associées à une bonne extensométrie à chaud permettent d'obtenir des résultats fiables, précis et reproductibles qui contribuent à la connaissance et à l'établissement des lois rhéologiques des matériaux.
\end{abstract}

\begin{abstract}
A creep machine with large stiffnesses aimed to perform high temperature biaxial tests at constant (tensile $\sigma_{z z}$ and shear $\sigma_{z \theta}$ ) stresses is described. The axial force is a dead weight one and the constancy of tensile stress is obtained through a cam which produces a motion of the force application point related to the sample tensile strain. The torsion torque is produced by torque motors, directly locked with the torsion grip, the current strength of which obeys the required control law. This device allows too cyclic shear stress tests with constant biasing tensile stress.

The possibilities of this machine together with an accurate high temperature strain measurements device give reliable and accurate results which take a part in the knowledge of the rheologic rules of materials.
\end{abstract}

\section{Intérêt de l'appareillage.}

L'étude des propriétés mécaniques des matériaux à haute température $\left(T / T_{\mathrm{F}} \geqq 0,3\right)$ sous sollicitations complexes, tant en régime stationnaire, transitoire que dynamique soulève encore de nombreux problèmes [1-6] et le cas de l'acier inoxydable 316 , où le couplage entre la plasticité dite «indépendante du temps » et le fluage à long terme, en constitue un bel exemple $[5,7]$.

Nous nous proposons présentement de décrire une nouvelle machine performante permettant de réaliser des essais de fluage en traction-torsion à haute température. Cette machine est asservie en contraintes compte tenu des grandes déformations que peuvent subir les matériaux au moment du chargement puis lors du fluage conduisant à la rupture par endommagement. Par exemple, dans le cas d'un acier du type 316 hypertrempé depuis $1200^{\circ} \mathrm{C}$, on obtient au chargement une déformation axiale $\varepsilon_{z z} \simeq 5 \%$ à $550{ }^{\circ} \mathrm{C}$ pour $\sigma_{z z}=300 \mathrm{MPa}$ conduisant à une vitesse d'écoulement quasiment stationnaire de l'ordre de $7 \times 10^{-10} \mathrm{~s}^{-1}$ au bout de $500 \mathrm{~h}$.

La nécessité d'analyser la réponse du matériau à des transitoires rapides (décrément brusque de contraintes) lors de l'établissement des lois d'écoulement [6-7] nous impose la solution d'une machine de bonne rigidité et d'une extensométrie précise et fiable. D'autre part, la solution électrodynamique retenue pour l'application du couple de torsion permet d'effectuer des essais de cyclage lent en torsion $(f \leqq 0,1 \mathrm{~Hz})$ sous contrainte axiale fixe, d'où la possibilité d'étudier l'effet de rochet associé au cyclage.

\section{Principe et réalisation de l'ensemble.}

2. 1 DÉFINITION DES ÉPROUVETTES. - Dans un essai de fluage biaxé de traction-torsion, on impose à l'éprou- 
vette des contraintes axiale et de cisaillement, $\sigma_{z z}$ et $\sigma_{z \theta}$, constantes et l'on mesure les déformations $\varepsilon_{z z}$, $\varepsilon_{z \theta}$ qui en résultent. La nature et la géométrie des éprouvettes associées à la dynamique de contrainte d'essai désirée conditionnent le dimensionnement de la machine. L'installation doit être conçue pour tester des aciers entre 350 et $750^{\circ} \mathrm{C}$, les contraintes maxima suivant chaque composante devant être de l'ordre de $500 \mathrm{MPa}$, ce qui donne une contrainte équivalente maximum $\bar{\sigma}$ de l'ordre de $1000 \mathrm{MPa}$. Compte tenu des possibilités d'obtention des matériaux (barre ou tôle épaisse) et de leur usinage, nous adoptons la forme de tube épais de $1 \mathrm{~mm}$ d'épaisseur pour un diamètre extérieur de $10 \mathrm{~mm}(e / r=0,2)$ et une longueur utile $l_{0}$ de $50 \mathrm{~mm}$ (éprouvette élancée $l_{0} / 2 r=5$ ) (Fig. 8). Les force et couple maximum appliqués à l'éprouvette sont donc respectivement de l'ordre de $F=1,4 \times 10^{4} \mathrm{~N}$ et $C=64 \mathrm{~N}$.m, ces deux grandeurs fixant les solutions technologiques à retenir.

Dans le cas des tubes épais, les contraintes $\sigma_{z z}$ et $\sigma_{z \theta}$ sont liées aux vitesses de déformations, $\dot{\varepsilon}_{z z}$ et $\dot{\varepsilon}_{z \theta}$, et évoluent suivant l'épaisseur du tube, le calcul des relations liant ces différentes grandeurs ainsi que leurs variations avec le rayon courant sont rapportés dans l'annexe $\mathrm{A}$.

2.2 LOIS D'ASSERVISSEMENT À RÉALISER. - Lors d'un essai de fluage uniaxial, il est bien connu que ce n'est pas la force mais la contrainte appliquée à l'éprouvette qu'il faut garder constante malgré son allongement. Il en est de même en régime biaxial où l'on doit réaliser $\sigma_{z z}$ et $\sigma_{z \theta}$ constantes. A l'aide des relations de conservations des volumes (volumes de l'éprouvette et du trou), on montre aisément que (A.3) peut s'écrire sous la forme :

$$
\frac{\sigma_{z z}}{F}=A\left(\frac{l}{l_{0}}\right) \text { et } \frac{\sigma_{z \theta}}{C}=B\left(\frac{l}{l_{0}}\right)^{3 / 2}
$$

qui se réduit à $\sigma_{z z} / F_{0}=A$ et $\sigma_{z \theta} / C_{0}=B$ pour $l=l_{0}$.

Les fonctions d'asservissement sont donc :

$$
\begin{aligned}
F \times l & =\text { Cte }=F_{0} l_{0} \\
C\left(\frac{l}{l_{0}}\right)^{3 / 2} & =\text { Cte }=C_{0} .
\end{aligned}
$$

2.3 OBTENTION DES CONTRAINTES ET RÉALISATION DE LEUR ASSERVISSEMENT. - Pour des essais statiques, le moyen le plus simple pour appliquer une force élevée à l'extrémité d'une éprouvette est d'amplifier la force créée par une charge morte (poids morts) à l'aide d'un système du type bras de levier. L'asservissement mécanique qui en résulte consiste donc à faire varier, avec l'allongement $\Delta l$ de l'éprouvette, le moment du point d'application de la charge morte par l'intermédiaire d'une came dont le profil est établi de manière à réaliser $F . l=C$ te, tout en respectant la géométrie du système. Le calcul de la came est donné dans l'annexe $\mathrm{B}$ associée à la figure 9. Le rapport initial du bras de levier est de 15 , ce qui donne, pour une charge maximum de $10^{3} \mathrm{~N}$, une force appliquée à l'éprouvette de $1,5 \times 10^{4} \mathrm{~N}$, soit une contrainte de l'ordre de $530 \mathrm{MPa}$. Notons que lors de la réalisation, il a été nécessaire de fixer de fines crémaillères sur la came et le support qui lui reste tangent de façon à éviter tout glissement, de même qu'un arceau de rappel fixé sur le bâti de la machine oblige le support des poids à rester vertical.

Pour appliquer le couple à l'éprouvette, nous avons adopté deux solutions; la première consiste comme précédemment à faire travailler des poids par l'intermédiaire d'un câble s'enroulant sur une poulie, l'asservissement mécanique n'étant pas possible dans cette situation (cas des petites déformations), alors que la seconde, purement électrodynamique, offre de nombreuses possibilités, entre autre celle de l'asservissement. Elle réside dans l'assemblage de deux moteurs couples à courant continu (type Artus T 7203) directement montés sur le mors de torsion relié à l'éprouvette, les pôles d'alimentation étant légèrement décalés d'un moteur à l'autre de manière à éviter l'ondulation du couple créée à chaque passage de pôle. L'avantage de cette solution réside dans le fait qu'il n'existe aucun jeu dans la transmission de l'effort lors de sollicitations dynamiques ou au passage à zéro lors de l'inversion du couple. D'autre part, les moteurs sont montés tête-bêche de façon à préserver l'accès aux balais, cè qui nécessite une inversion de polarité d'un moteur à l'autre. Chaque moteur, dont la résistance interne est de $0,88 \Omega$, délivre un couple maximum de $30,7 \mathrm{Nm}$ pour une intensité crête de $24,5 \mathrm{~A}$. Les deux moteurs sont alimentés en série, ce qui donne un couple maximum disponible de l'ordre de $61 \mathrm{~N} . \mathrm{m}$ pour l'intensité crête (soit $\sigma_{z \theta} \simeq 480 \mathrm{MPa}$ ) et une sensibilité de 2,50 Nm/A.

L'intensité fournie aux moteurs est délivrée par une alimentation de puissance unipolaire commutable

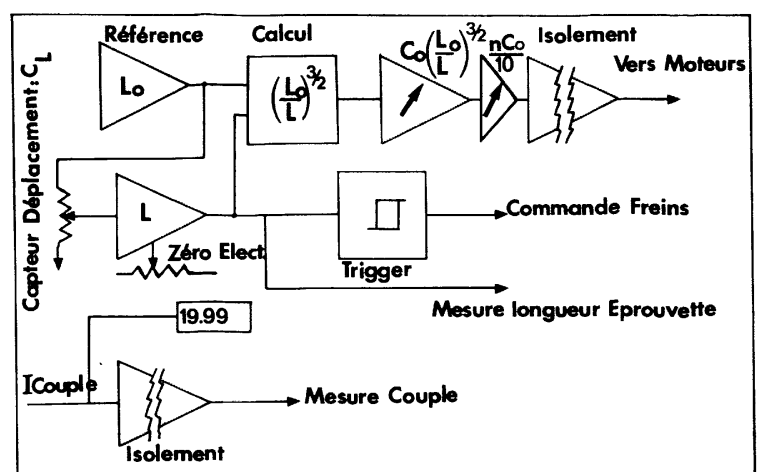

Fig. 1. - Synoptique de l'électronique d'asservissement des moteurs couples.

[Synoptic diagram of the electronics for torque motors servo control.] 
du type "Varian " dont la puissance disponible est de $2000 \mathrm{~W}$ sous $0,5 \Omega$, la puissance maximum utilisée n'est que de $1100 \mathrm{~W}$. Le signal de sortie de cet amplificateur de puissance peut être commandé de trois façons différentes :

- manuellement à l'aide d'un potentiomètre dix tours,

- en balayage automatique interne à l'aide de moteurs de commande installés sur l'alimentation,

- en mode externe par l'intermédiaire d'un module de commande.

C'est par ce pilotage externe qu'est réalisé l'asservissement de l'intensité $I$ parcourant les moteurs couples, soit $I=I_{0}\left(l_{0} / l\right)^{3 / 2}$ avec $I_{0}$ variable (cf. Eq. 2).

Le schéma synoptique de la réalisation de cette loi, et dont le principe est le suivant, est donné sur la figure 1 : un capteur de déplacement résistif $C_{\mathrm{L}}$ $(10 \mathrm{k} \Omega, 100 \mathrm{~mm}, \pm 0,1 \%$ ), monté dans le prolongement de l'éprouvette et alimenté par une tension de référence, délivre un signal proportionnel à la longueur $l$ de l'éprouvette, le zéro électrique ayant été préalablement réglé en début d'essais. Un autre signal proportionnel à la longueur $l_{0}$ est délivré par un régulateur de tension. Ces deux signaux sont ensuite appliqués à un opérateur multifonction (Teledyne 4371) qui a été calé sur table pour réaliser la fonction $S_{0}=Y(Z / X)^{m}$ avec $Y=1, Z=l_{0}, X=l$ et $m=3 / 2$ et le signal résultant est de nouveau appliqué à un amplificateur de gain $N$ fixant la valeur initiale de $I_{0}$ (ou $C_{0}$ ). Un étage supplémentaire de gains discrets commutables $(n / 10$ avec $0 \leqq n \leqq 10)$ permet de réaliser des décréments rapides d'intensité, simulant des sauts de contraintes. La liaison vers l'amplificateur de puissance se fait par l'intermédiaire d'un amplificateur d'isolement de gain unité, les entrées de l'amplificateur de puissance étant référencées par rapport à un zéro électrique isolé de la masse. La fonction effectuée à l'aide de ce montage a été testée sur table et approche la loi réelle à $1 \%$ près.

De manière à arrêter la rotation du mors pouvant entraîner l'endommagement des extensomètres, lors de la rupture de l'éprouvette, un frein électromagnétique $F_{r}$, piloté par un trigger, est rendu solidaire de l'arbre de torsion. En effet, lorsque la vitesse de déformation de l'éprouvette donnée par le capteur de déplacement dépasse une valeur fixée, le trigger, pilotant le relais d'alimentation du module de puissance du frein, autorise le serrage de celui-ci. Il en est de même pour la charge axiale où un frein à disque $F_{\mathrm{a}}$ permet le bloquage du mors supérieur.

2.4 Réalisation méCANiQue DE L'ENSEMBLE. - Les figures 2 et 3 donnent respectivement un plan mécanique d'ensemble de la machine (éch. 1/35) et un schéma synoptique de l'infrastructure électrique et électronique qui lui est associée. La photo 4 représente une vue d'ensemble de la machine.

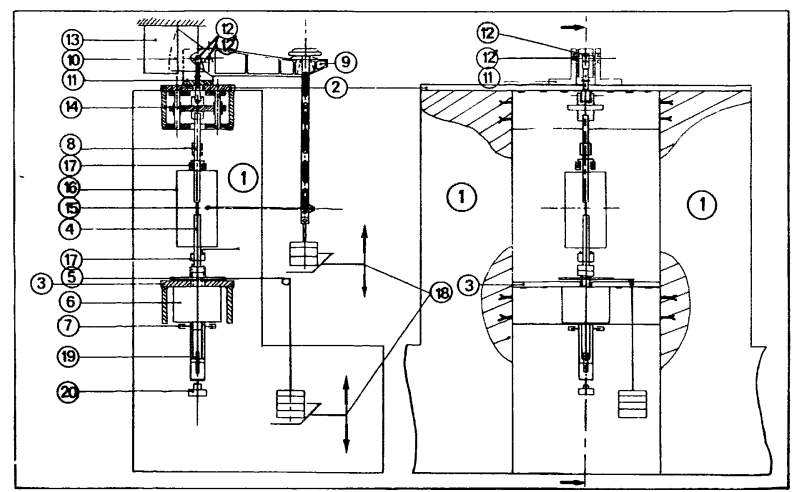

Fig. 2. - Schéma mécanique de la machine (éch. $1 / 10 \times 1 / 3,5)$.

[Mechanical diagram of the machine (scale $1 / 10 \times 1 / 3.5$ ). $]$

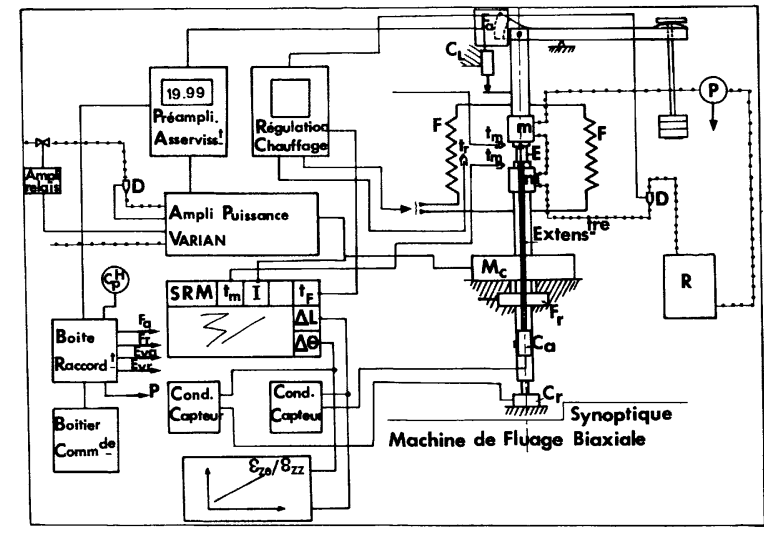

Fig. 3. - Synoptique d'ensemble (mécanique et électronique) de la machine.

[Comprehensive synoptic diagram (mechanics and electronics) of the machine.]

De façon à obtenir un ensemble rigide et dimensionnellement stable (problème de stabilité thermique), le bâti est composé de deux piliers verticaux en béton (1) dans lesquels sont scellées deux platines de référence en acier soudé $(30 \mathrm{~mm}$ d'épais.) et de profil en $\sqcap$, (2) et (3). La platine inférieure supporte, par l'intermédiaire d'une butée à double effets préalablement tarée, le mors de torsion (4) solidaire des poulies de la charge morte (5) et des parties tournantes des moteurs couple (6) et du frein radial (7).

La platine supérieure sert de référence au mors mobile de translation (8) et supporte la came (9) et son équilibrage (10). Ce mors est rendu solidaire de la came grâce à un axe (12) monté sur roulement à billes, la came pouvant elle-même pivoter par rapport à son point d'appui matérialisé par un autre axe (12), également muni de roulement à billes et supporté par deux étriers (11). La distance entre axes définit la longueur $" b »$ du bras de levier (Fig. 9). Le frein à disque axial (13) est fixé au plafond de la salle et agit sur des surfaces rectifiées de la masse d'équilibrage. 


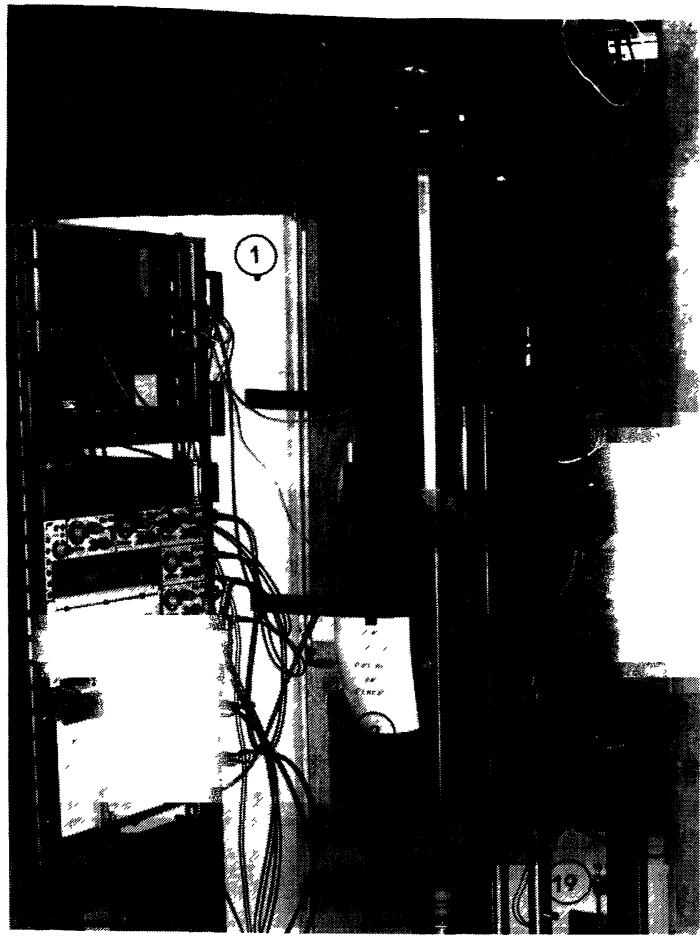

Fig. 4. - Photographie d'ensemble de la réalisation.

[Photograph of the whole device.]

Les mouvements de translation et de rotation sont découplés par une colonne de guidages à billes (14). dont l'efficacité s'avère satisfaisante. En effet, les modules d'Young et de Coulomb de l'éprouvette (15), mesurés à l'aide de jauges de contrainte, respectivement en présence de différentes charges radiales et axiales, n'évoluent que de $3 \%$, pour des charges correspondant sensiblement à la moitié de la capacité de la machine (§ 2.3). La raideur longitudinale de l'ensemble est d'environ $9 \times 10^{4} \mathrm{~N}$. mm.

L'ensemble est équipé d'un four ouvrant (16), type Adamel à régulation P.I.D. et permet de travailler de 300 à $900^{\circ} \mathrm{C}$. Les mors sont refroidis à la sortie du four par un circuit fermé (17) dans lequel circule de l'eau distillée provenant d'une réserve.

Notons également qu'il est possible d'effectuer des allègements brusques et synchronisés de charges ( " dip test technique " $[10,6])$ par l'intermédiaire de vérins pneumatiques (18) commandés par des électrovannes, possibilité déjà mentionnée dans le cas des moteurs couples $(\S 2.3)$.

\subsection{EXTENSOMÉTRIE ET ACQUISITION DES INFORMATIONS.} La géométrie tubulaire de l'éprouvette est exploitée pour réaliser une extensométrie longitudinale très précise (Fig. 5). Celle-ci est obtenue à l'aide de deux tiges concentriques en acier inoxydable (a), (b), la plus fine (a) venant s'appuyer sur le cône de centrage du fond du trou de l'éprouvette, la tige extérieure (b) s'appuyant sur un siège usiné dans la tête opposée de cette dernière (Figs. 5 et 8 ). Cette solution permet

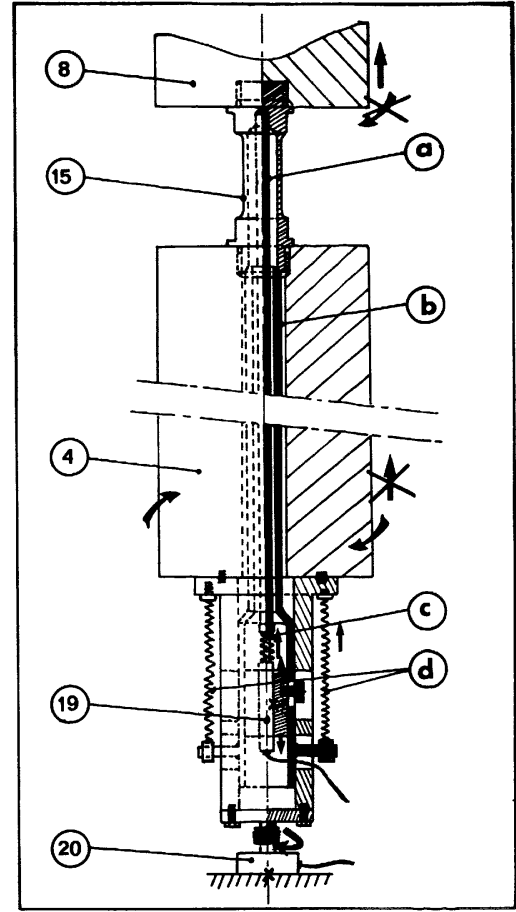

Fig. 5. - Schéma du montage extensomètre.

[Extensometric device.]

une mesure fine de l'allongement effectif du corps de l'éprouvette tout en permettant la localisation du capteur (19) à l'extérieur de la zone chaude et dans l'axe du mors de rotation (Figs. 2, 3, 5). Les positionnements des tiges intérieure et extérieure sont respectivement assurés par la poussée du doigt (c) du capteur (19) et par des ressorts (d) solidaires du mors de torsion (Fig. 5).

Le rotor du capteur rotatif (20) est directement monté en bout de l'arbre de torsion, le stator étant référencé au bâti de la machine (Fig. 5). Compte tenu de la précision du guidage au niveau des colonnes de (14), celui-ci ne rapportant aucun jeu, la précision obtenue est bonne (exemple Fig. 6). On peut noter de plus qu'aucun effet parasite n'a été mis en évidence lors du passage à zéro dans une expérience d'inversion de couple.

Les capteurs (19) et (20) sont respectivement des transformateurs différentiels linéaires (L.V.D.T., « Enertec ») et rotatif (R.V.D.T., "Shaewitz ») dont les caractéristiques sont les suivantes : étendue de mesure $\pm 2,5 \mathrm{~mm}, \pm 60^{\circ}$, linéarité : $0,1 \%$ et $0,5 \%$, sensibilité : $150 \mathrm{mV} / \mathrm{V}$. alim. $/ \mathrm{mm}$ et $2,8 \mathrm{mV} /$ degré $/ \mathrm{V}$. alim. pour une porteuse dont la fréquence est de $5 \mathrm{~Hz}$ pour un niveau de $5 \mathrm{~V}$, la résolution obtenue étant pratiquement infinie. Chaque capteur est relié à un conditionneur universel "Enertec" (capteur différentiel, jauge de contrainte, ...), à gain variable et à décalage de niveau de sortie, qui restitue un signal de sortie continue proportionnel au déplacement enregistré par le capteur. 


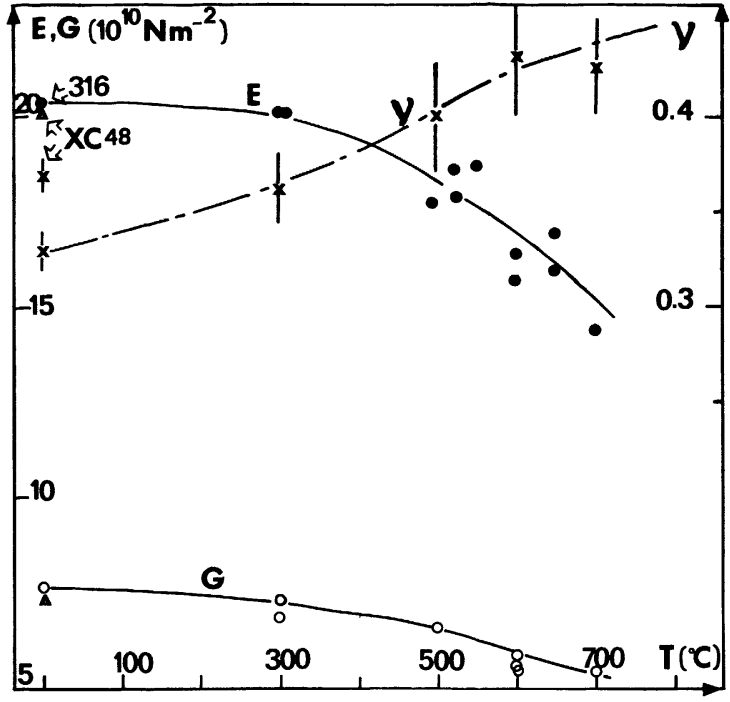

Fig. 6. - Mesure statique des constantes élastiques de deux aciers.

[Static measurement of the elastic constants of two steels.]

Toutes les informations, températures d'essais $t_{\mathrm{m}}$ et de régulation $t_{\mathrm{f}}$, intensité parcourant les moteurs couple $I$, allongement $\Delta l$ et rotation $\Delta \theta$ de l'éprouvette, sont simultanément collectées sur un enregistreur rapide à six voies synchrones (Sefram). Notons qu'une acquisition de données rapide par micro-calculateur (type "Commodor ») est en cours d'installation. Une table traçante calibrée permet d'obtenir en continu, directement la direction d'écoulement $\varepsilon_{z \theta} / \varepsilon_{z z}=$ $r \Delta \theta / 2 \Delta l$ (exemple Fig. 7).

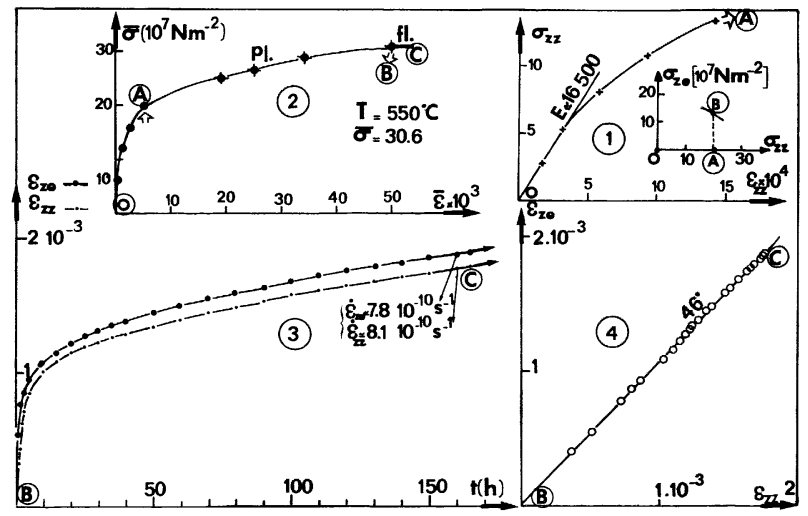

Fig. 7. - Exemple de résultats complexes obtenus sur un acier du type 316 à $550^{\circ} \mathrm{C}$.

[Examples of results got on a 316 stainless steel at $550^{\circ} \mathrm{C}$.]

\section{Présentation de quelques résultats obtenus sur un acier inoxydable 316.}

Les éprouvettes tubulaires sont obtenues à partir de lopins découpés mécaniquement dans une tôle de
$30 \mathrm{~mm}$ d'épaisseur, hypertrempée depuis $1200^{\circ} \mathrm{C}$. Les opérations de cylindrage, perçage et mise aux cotes sont effectuées par fines passes successives $(\simeq 0,2 \mathrm{~mm})$ de façon à minimiser l'évenţuel écrouissage introduit par l'usinage, aucun traitement thermique ultérieur n'étant réalisé de façon à préserver la bonne géométrie de l'éprouvette.

La figure 6 donne un exemple de détermination en fonction de la température, des constantes élastiques macroscopiques obtenues à partir de l'extensométrie précédemment décrite :

$$
E=\frac{\Delta F l_{0}}{\pi\left(r_{2}^{2}-r_{1}^{2}\right) \Delta l}, \quad G=\frac{2 l_{0} \Delta C}{\pi\left(r_{2}^{4}-r_{1}^{4}\right) \Delta \theta} .
$$

Les valeurs du coefficient de Poisson $v$ déduites de la relation $v=\left(\frac{E}{2 G}-1\right)$, sont tout à fait acceptables. En effet, ce coefficient croît de 0,33 à l'ambiante à environ 0,42 pour $T \simeq 700^{\circ} \mathrm{C}(v \rightarrow 0,5$ dans le cas d'un corps plastique parfaitement incompressible). Les valeurs calculées à l'ambiante, par l'intermédiaire de jauges de contrainte, ne diffèrent que d'environ $5 \%$ de celles présentement mesurées.

La figure 7 donne, à travers l'exemple du comportement sous sollicitation biaxial d'un acier 316 à $550^{\circ} \mathrm{C}$, un aperçu des performances de la machine. Dans le plan des contraintes (Fig. 7.1), la sécance expérimentale se résume de la manière suivante : chargement axial de manière incrémentale suivant $\mathbf{O A}$, croissance linéaire de la contrainte de cisaillement suivant $\mathrm{AB}$ puis maintien au point de fonctionnement $B$. Lors du chargement axial OA, on mesure la déformation élastique suivie d'une déformation viscoplastique rapide ou plasticité dite "indépendante du temps ». On peut remarquer la très faible limite élastique de cet acier. Lorsque la contrainte $\sigma_{z \theta}$ passe de A en B à vitesse linéaire constante, on assiste, selon chaque composante, à de grandes déformations hétérogènes et rapides $\left(\bar{\varepsilon}=\left\{\varepsilon_{z z}^{2}+4 / 3 \varepsilon_{z \theta}^{2}\right\}^{1 / 2} \simeq 4 \times\right.$ $10^{-2}$, partie $\mathrm{AB}$ de 7.2). Les contraintes étant maintenues au point $B$, on mesure ensuite les composantes du fluage (partie $\mathrm{BC}$ de 7.2, 7.3). Ce matériau présente une très forte consolidation qui conduit à des vitesses faibles de l'ordre de $8 \times 10^{-10} \mathrm{~s}^{-1}$ au bout de $170 \mathrm{~h}$. On peut remarquer la précision et la stabilité de l'extensométrie sur les très faibles déformations, par exemple au bout de $120 \mathrm{~h}$ chaque point espacé de $10 \mathrm{~h}$ correspond sensiblement à des incréments de déformations $\varepsilon_{z z} \simeq \varepsilon_{z \theta} \simeq 4 \times 10^{-5}$ soit $2 \mu \mathrm{m}$ d'allongement et $3^{\prime}$ d'arc de rotation. Ces deux facteurs se retrouvent également sur la vérification quasi parfaite de la règle de normalité de l'écoulement de VonMisès [6-7] (Fig. 7.4) :

$$
\frac{\dot{\varepsilon}_{z z}}{\dot{\varepsilon}_{z \theta}}=\frac{\varepsilon_{z z}}{\varepsilon_{z \theta}}=\frac{2}{3} \frac{\sigma_{z z}}{\sigma_{z \theta}} .
$$

On voit l'intérêt de cette machine pour l'étude des matériaux présentant des comportements aussi 
complexes que ceux de l'acier 316 , à savoir, grandes déformations très rapides suivies de petites déformations lentes. Dans l'exemple de la figure 7, on enregistre environ sept décades de vitesse en $170 \mathrm{~h}$.

\section{Conclusions.}

Nous avons présenté la réalisation d'une machine de fluage biaxiale asservie (traction-torsion) de grande rigidité permettant l'étude des lois de comportement des aciers, à haute température et sous sollicitations complexes.

Les solutions retenues pour l'application des forces et couples sur l'éprouvette permettent également d'effectuer des cyclages lents en torsion sous contrainte axiale fixe.

La bonne rigidité de l'ensemble associée à une extensométrie précise permet l'étude fine des transitoires rapides (décréments et incréments brusques de contraintes).

\section{Remerciements.}

Les auteurs tiennent particulièrement à remercier Messieurs R. Cosme et G. Laithier, techniciens au C.N.R.S. pour leur participation à la réalisation et à la mise au point de cet appareillage.

\section{ANNEXES}

\section{Annexe A.}

Calcul des relations liant les contraintes $\sigma_{z z}, \sigma_{z \theta}$ aux vitesses de déformation $\dot{\varepsilon}_{z z}, \dot{\varepsilon}_{z \theta}$ dans le cas d'un tube épais.

Pour traiter ce problème, nous utilisons la méthode de Hill [8] qui conduit à considérer un tube épais comme un assemblage concentrique de tubes minces dans chacun desquels l'état de contrainte et de déformation se développe indépendamment. Si l'on se place en coordonnées cylindriques $r, \theta, z$ et si les vitesses de déformation sont uniquement fonction du rayon courant $r$, l'extension des lois de la plasticité $\left(\varepsilon_{i j}=\lambda \partial f / \partial \sigma_{i j} f\right.$ étant le potentiel plastique) à la viscoplasticité (existence d'un potentiel viscoplastique $\Omega$ tel que $\dot{\varepsilon}_{i j}=\partial \Omega / \partial \sigma_{i j}$ ), conduit aux relations (A.1) [9] :

$$
\begin{aligned}
& \dot{\varepsilon}_{r r}=-\frac{i}{2 l}=K(T) \bar{\sigma}^{n-1} S_{r r} \\
& \dot{\varepsilon}_{\theta \theta}=-\frac{i}{2 l}=K(T) \bar{\sigma}^{n-1} S_{\theta \theta} \\
& \dot{\varepsilon}_{z z}=\frac{i}{l}=K(T) \bar{\sigma}^{n-1} S_{z z} \\
& \dot{\varepsilon}_{z \theta}=\frac{\dot{\gamma}}{2}=\frac{r \dot{\theta}}{2 l}=K(T) \bar{\sigma}^{n-1} S_{z \theta} \\
& \dot{\varepsilon}_{r z}=\dot{\varepsilon}_{r \theta}=0 .
\end{aligned}
$$

Dans ces équations, $l$ est la longueur de l'éprouvette à l'instant considéré et $\theta$ l'angle de torsion subi par celle-ci, $K(T)$ est une constante thermiquement activée, $n$ le coefficient de sensibilité de la vitesse à la contrainte du matériau, $S_{i j}$ les composantes du déviateur des contraintes et $\bar{\sigma}$ la contrainte équivalente de Von-Misès donnée par $\bar{\sigma}=\left\{\sigma_{z z}^{2}+3 \sigma_{z \theta}^{2}\right\}^{1 / 2}$. Dans le cas de l'anisotropie de Hill [8], repère d'anisotropie identique au repère $r, \theta, z$, la contrainte équivalente est donnée par $\bar{\sigma}=\left\{\sigma_{z z}^{2}+\beta \sigma_{z \theta}^{2}\right\}^{1 / 2}$ avec $\beta=3 L / N$, $L$ et $N$ étant deux constantes d'anisotropie.

Les relations intégrales (A.2) liant la force $F$ à la contrainte axiale $\sigma_{z z}$ et le couple $C$ à la contrainte tangentielle $\sigma_{z \theta}$ conduisent aux rapports $\sigma_{z z} / F$ et $\sigma_{z \theta} / C$ donnés par (A.3).

$$
\begin{aligned}
& \left.\begin{array}{l}
F=\int_{r_{1}}^{r_{2}} 2 \pi r \sigma_{z z} \mathrm{~d} r \\
C=\int_{r_{1}}^{r_{2}} 2 \pi r^{2} \sigma_{z \theta} \mathrm{d} r
\end{array}\right\} \\
& \frac{\sigma_{z z}}{F}=\frac{n+1}{2 n} \cdot\left(\frac{\dot{\gamma}}{r}\right)^{2} \cdot\left(\frac{\left(\gamma^{2}+\beta \dot{\varepsilon}_{z z}^{2}\right)^{\frac{1-n}{2 n}}}{\left(\dot{\gamma}^{2}\left(r_{2} / r\right)^{2}+\beta \dot{\varepsilon}_{z z}^{2}\right)^{\frac{1+n}{2 n}}-\left(\dot{\gamma}^{2}\left(r_{1} / r\right)^{2}+\beta \dot{\varepsilon}_{z z}^{2}\right)^{\frac{1+n}{2 n}}}\right) \\
& \frac{\sigma_{z \theta}}{C}=\frac{3 n+1}{2 n} \cdot\left(\frac{\dot{\gamma}^{4}}{r^{3}}\right) \times \\
& \times\left(\frac{\left(\gamma^{2}+\beta \dot{\varepsilon}_{z z}^{2}\right)^{\frac{1-n}{2 n}}}{\left[\left[\dot{\gamma}^{2}\left(r_{2} / r\right)^{2}+\beta \dot{\varepsilon}_{z z}^{2}\right]^{\frac{n+1}{2 n}} \cdot\left[\dot{\gamma}^{2}\left(r_{2} / r\right)^{2}-\frac{2 n \beta \dot{\varepsilon}_{z z}^{2}}{n+1}\right]\right]-\left[\left[\dot{\gamma}^{2}\left(r_{1} / r\right)^{2}+\beta \dot{\varepsilon}_{z z}^{2}\right]^{\frac{n+1}{2 n}} \cdot\left[\gamma^{2}\left(r_{1} / r\right)^{2}-\frac{2 n \beta \dot{\varepsilon}_{z z}^{2}}{n+1}\right]\right.}\right) .
\end{aligned}
$$

Nous avons fait varier $\beta$ de 1 à 5 , les autres paramètres étant fixés et l'on constate que les rapports $\sigma_{z z} / F$ et $\sigma_{z \theta} / C$ sont très peu sensibles à $\beta$ (variation inférieure à $4 \%$ ), c'est-à-dire que les éventuels effets d'anisotropie 
visibles sur la loi de normalité ; $\frac{\dot{\varepsilon}_{z z}}{\dot{\varepsilon}_{z \theta}}=\frac{2}{3} \frac{N}{L} \frac{\sigma_{z z}}{\sigma_{z \theta}}$, ne seront pas trop affectés par le calcul des contraintes en prenant $\beta=3$ (contrainte de Von-Misès) dans (A.3).

Pour nos éprouvettes, l'évolution de ces rapports en fonction du rayon courant $r$, dans différentes situations de vitesses, est représentée sur la figure 8. On constate

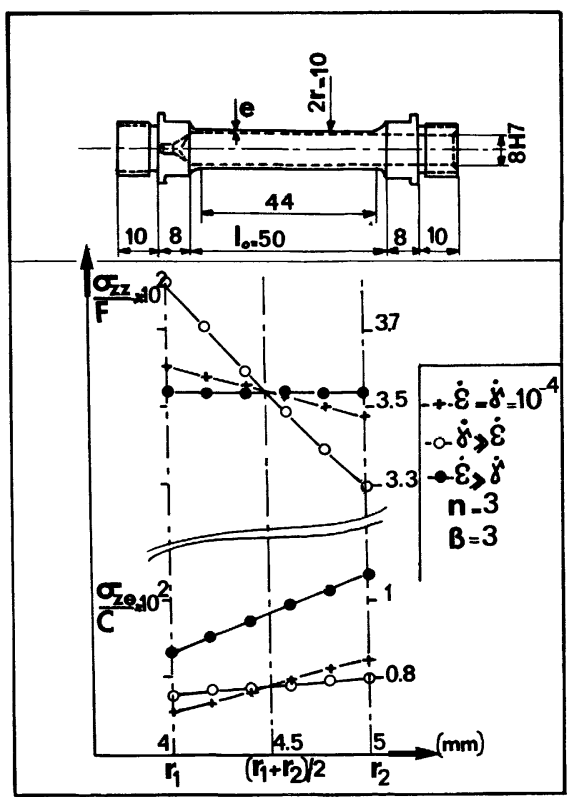

Fig. 8. - Caractéristiques des éprouvettes.

[Characteristics of the samples.]

que le cas de chargements très dissymétriques, où l'une des vitesses est très supérieure à l'autre, conduit aux plus grandes inhomogénéités sur les contraintes. Ainsi, dans le cas proche de la traction $\dot{\varepsilon}_{z z} \gg \dot{\gamma}, \sigma_{z \theta} / C$ croît de $20 \%$ avec le rayon courant alors que $\sigma_{z z} / F$ est constant. Dans le cas inverse $\dot{\gamma} \gg \dot{\varepsilon}_{z z}, \sigma_{z z} / F$ décroît de $16 \%$ alors que simultanément, $\sigma_{z \theta} / C$ augmente de $7 \%$ avec $r$. Pour des vitesses du même ordre de grandeur, par exemple $\dot{\varepsilon}_{z z}=\dot{\gamma}=10^{-4} \mathrm{~s}^{-1}$, on obtient respectivement sur $\sigma_{z \theta} / C$ et $\sigma_{z z} / F$ des variations de $16 \%$ et $3,5 \%$.

Cependant, pour un calcul rapide et souvent suffisant, une bonne approximation est fournie par (A.2) en supposant $\sigma_{z z}$ et $\sigma_{z \theta}$ constants.

$$
\left.\begin{array}{c}
\sigma_{z z}=\frac{F}{\pi\left(r_{2}^{2}-r_{1}^{2}\right)} \\
\sigma_{z \theta}=\frac{3 C}{2 \pi\left(r_{2}^{3}-r_{1}^{3}\right)}
\end{array}\right\} .
$$

\section{Annexe B.}

Etablissement du profil de la came (Fig. 9). - A l'aide de l'hypothèse de la déformation de l'éprouvette à

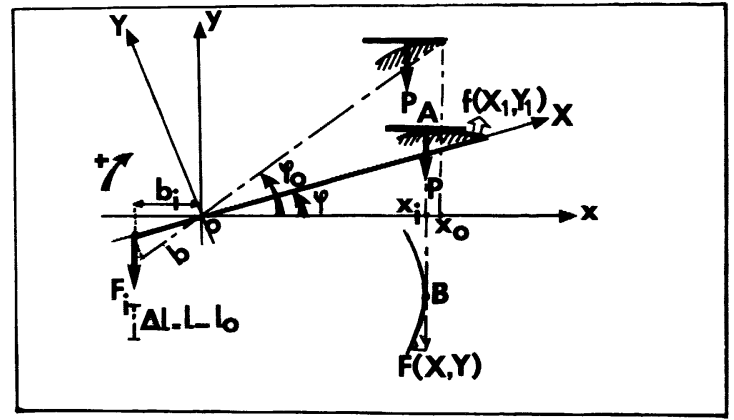

Fig. 9. - Eléments de calcul de la came.

[Cam calculation generalities.]

volume constant, on montre que la première équation de (A.3) s'écrit :

$$
\frac{\sigma_{z z}}{F}=A\left(\frac{l}{l_{0}}\right) .
$$

La loi d'asservissement axiale à réaliser est donc :

$$
F . l=F_{0} \cdot l_{0}=\text { Cte }
$$

l'indice zéro correspondant à l'état non déformé de l'éprouvette.

Si l'on tient compte de l'équation d'égalité des moments, $x_{i} P=b_{i} F$, on arrive à exprimer $x_{i}$ en fonction de l'angle de rotation $\varphi$ du bras de levier :

$$
x_{i}=\frac{l_{0} x_{0}}{\cos \varphi_{0}}\left(\frac{\cos \varphi}{l_{0}+b\left(\sin \varphi-\sin \varphi_{0}\right)}\right) .
$$

Les caractéristiques mécaniques sont telles que : $l_{0}=50 \mathrm{~mm}, b=40 \mathrm{~mm}, x_{0}=600 \mathrm{~mm}$ (le rapport du bras de levier est donc $x_{0} / b=15$ ), l'angle pouvant varier de -15 à $+15^{\circ}$, ce qui correspond à un allongement de l'éprouvette de $20 \mathrm{~mm}$, soit $40 \%$.

$$
\text { Posons } \quad B=\frac{l_{0}}{b}-\sin \varphi_{0} \quad \text { et } \quad A=\frac{l_{0} x_{0}}{b \cos \varphi_{0}},
$$

on obtient $x_{i}=A \frac{\cos \varphi}{B+\sin \varphi}$.

Dans le repère $\{X O Y\}$, l'enveloppe des normales à la came $F(X, Y)$ (droites $\mathrm{AB})$ est telle que :

$$
\left.\begin{array}{l}
X=\frac{A}{B+\sin \varphi}\left[\cos ^{2} \varphi+\frac{\sin \varphi(B \sin \varphi+1)}{B+\sin \varphi}\right] \\
Y=\frac{A \cos \varphi}{B+\sin \varphi}\left[\sin \varphi-\frac{B \sin \varphi+1}{B+\sin \varphi}\right]
\end{array}\right\}
$$

et aux petits angles :

$$
\left.\begin{array}{l}
X=\frac{A(B+2 \varphi)}{(B+\varphi)^{2}} \\
Y=\frac{-A}{(B+\varphi)^{2}}
\end{array}\right\} .
$$



L'équation de la came $f\left(X_{1}, Y_{1}\right)$ n'est autre que la initiale $\varphi=\varphi_{0}=-15^{\circ}, X_{10}=\frac{x}{\cos \varphi_{0}}$ et $Y_{10}=0$,
développante de $F(X, Y)$ correspondant à la condition

soit :

$$
\left.\begin{array}{l}
X_{1}=\frac{A(B+2 \varphi)}{(B+\varphi)^{2}}-\frac{\varphi}{\sqrt{1+\varphi^{2}}}\left[k-2 A \int \frac{\sqrt{1+\varphi^{2}}}{(B+\varphi)^{3}} \mathrm{~d} \varphi\right] \\
Y_{1}=\frac{-A}{(B+\varphi)^{2}}+\frac{1}{\sqrt{1+\varphi^{2}}}\left[k-2 A \int \frac{\sqrt{1+\varphi^{2}}}{(B+\varphi)^{3}} \mathrm{~d} \varphi\right]
\end{array}\right\}
$$

Le système (B.6) n'admet pas de solution analytique simple, mais un développement aux petits angles, $\left(\sqrt{1+\varphi^{2}} \simeq 1+\frac{\varphi^{2}}{2}\right)$, conduit à la solution paramétrique approchée de $f\left(X_{1}, Y_{1}\right)$ :

$$
\left.\begin{array}{l}
\left.X_{1}=\frac{A(B+2 \varphi)}{(B+\varphi)^{2}}-\varphi\left(1-\frac{\varphi^{2}}{2}\right)\left[k-A\left(\frac{2 B}{B+\varphi}-\frac{1}{(B+\varphi)^{2}}\left(1+\frac{B^{2}}{2}\right)+\operatorname{Ln}|B+\varphi|\right)\right]\right) \\
Y_{1}=-\frac{A}{(B+\varphi)^{2}}+\left(1-\frac{\varphi^{2}}{2}\right)\left[k-A\left(\frac{2 B}{B+\varphi}-\frac{1}{(B+\varphi)^{2}}\left(1+\frac{B^{2}}{2}\right)+\operatorname{Ln}|B+\varphi|\right)\right]
\end{array}\right\} .
$$

La condition initiale $Y_{10}=0$ pour $\varphi_{0}=-15^{\circ}$ donne $k=1513 \mathrm{~mm}$.

Nous avons également tracé géométriquement la développante de la solution exacte de $F(X, Y)(\mathrm{B} .4)$, la courbe ainsi obtenue est identique à la représentation de (B.7) et justifie a posteriori les approximations successives prises dans le calcul. La précision de la réalisation mécanique de l'ensemble approche la loi (B.2) à $2 \%$ près.

\section{Bibliographie}

[1] Krempl, E., Weld. Res. Counc. Bull. 195 (1974) 63.

[2] Phillips, A., The foundations of plasticity ICMS, Udine (1974).

[3] Rice, J. R. in A. S. Argon (ed.). Constitutive Equations in Plasticity (Massachusetts Institute of Technology Press, Cambridge, MA.) 1975, p. 29-75.

[4] Findley, W. N., Cho, U. W., Ding, J. L., J. Eng. Mat. Techn. 101 (1979) 365.

[5] Chaboche, J. L., Rousselier, G., 3rd International Seminar SMIRT 6, Paris 24-25 Août (1981).
[6] Oytana, C., Delobelle, P., Mermet, A., J. Eng. Mat. Techn. 104 (1982) 1.

[7] Delobelle, P., Oytana, C., Mermet, A., à paraître, ICM IV, Stockholm Sweden 15-19 August (1983).

[8] Hill, R., The mathematical theory of plasticity 1964 (Oxford, University Press, London) 1964.

[9] Delobelle, P., Oytana, C., Mermet, A., Revue Phys. Appl. 14 (1979) 933.

[10] Ahlquist, C. N., Nix, W. D., Scripta. Metall. 3 (1969) 679. 